Revista Thema

\begin{tabular}{l|l|l|} 
v.19 & n.2 & 2021
\end{tabular} p.456-470

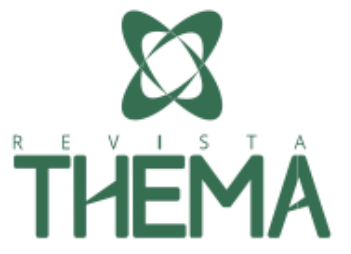

\section{A relação entre a idade e o "momento competitivo" com os níveis de coesão de grupo em atletas de Basquetebol}

\author{
The relationship between age and the "competitive moment" \\ with the levels of group cohesion in Basketball athletes \\ Douglas Versuti Arantes Alvarenga ${ }^{1}$, \\ Guilherme Henrique Cordeiro Xavier², Bruna Maria Genuíno Sousa ${ }^{3}$, \\ Bruna Alves Santana ${ }^{4}$, Rosana Lopes da Silva Garcia ${ }^{5}$, \\ Ivan Wallan Tertuliano ${ }^{6}$
}

\title{
RESUMO
}

O presente estudo objetivou investigar a relação entre a idade (sub-13 e sub-15) e o momento competitivo (início e final da competição) com os níveis de coesão de grupo em atletas amadores de basquetebol. Participaram 26 atletas (12 e 14 respectivamente). 0 instrumento utilizado foi o Questionário de Ambiente de Grupo: GEQ. As análises foram conduzidas entre os grupos, comparando os resultados do início e do final do campeonato. Utilizou-se o teste U de Mann-Whitney para comparação entre grupos, e o Friedman para análise intragrupo. Os resultados demonstraram que não houve diferença significante entre os grupos em nenhum momento do campeonato (início e final), para nenhuma dimensão, com os grupos investigados apresentando elevados escores nas dimensões relacionadas à tarefa, tanto no início quanto no final do campeonato. Assim, pode-se considerar que nem a idade, nem o momento da competição influenciaram os níveis de coesão, pois os dois grupos do estudo apresentaram níveis de coesão similares e elevados, tanto no início quanto no final da competição, o que é apontado como relevante para o bom desempenho esportivo dos atletas.

Palavras-chave: Coesão de grupo; basquetebol; psicologia do esporte; ambiente de grupo.

\section{ABSTRACT}

The present study aimed to investigate the relationship between age (under-13 and under-15) and the competitive moment (beginning and end of competition) with the levels of group cohesion in amateur basketball athletes. 26 athletes participated (12 and 14 respectively). The instrument used was the Group Environment Questionnaire - GEQ. The analyzes were

\footnotetext{
${ }^{1}$ Centro Universitário Adventista de São Paulo - UNASP, São Paulo/SP - Brasil. E-mail: doalvarenga10@ gmail.com

2 Idem. E-mail: guilherme edfisica@outlook.com

${ }^{3}$ Idem. E-mail: brunagenuino31@gmail.com

${ }^{4}$ Idem. E-mail: brunnasantna@gmail.com

${ }^{5}$ Centro Universitário Ítalo Brasileiro - Ítalo, São Paulo/SP - Brasil. E-mail: robask2906@gmail.com

6 Escola de Ciências da Saúde, Universidade Anhembi Morumbi, São Paulo/SP - Brasil. E-mail: ivanwallan@gmail.com
} 
conducted between the groups, comparing the results of the beginning and the end of the championship. Mann-Whitney $U$ test was used for comparison between groups, and Friedman for intra-group analysis. The results showed that there was no significant difference between the groups at any time of the championship (beginning and end), for any dimension, with the investigated groups presenting high scores in the dimensions related to the task, both at the beginning and at the end of the championship. Thus, it can be considered that neither age nor the moment of competition factor influenced the levels of cohesion, since the two groups in the study had similar and high levels of group cohesion, both at the beginning and at the end of the competition, which is considered relevant to the good sporting performance of athletes.

Keywords: Group cohesion; basketball; psychology of sport; group environment.

\section{INTRODUÇÃO}

De acordo com a literatura, coesão refere-se à união de um grupo para alcançar metas e, além disso, a busca pela satisfação dos membros a determinados objetivos e/ou tarefas em conjunto. (WEINBERG; GOULD, 2017). Assim, tal temática apresenta-se relevante para o esporte, haja vista que no esporte essa relação grupal pode influenciar os resultados dos atletas. (ASAMOAH; GROBBELAAR, 2017; NASCIMENTO JUNIOR et al., 2016a; NASCIMENTO JÚNIOR et al., 2018; SILVEIRA; OLIVEIRA, 2017; TERTULIANO et al., 2019; XAVIER et al., 2020).

Almeida (2008) expõe que o termo coesão de grupo foi inicialmente aplicado por Kurt Lewin e seus colaboradores em 1945, onde o propósito de um grupo consiste, maioritariamente, na interdependência entre os seus membros, e não somente na sua semelhança, sendo que a interdependência interpessoal é o que determina o grau de coesão de um grupo. De acordo com Mikalachki (1969), a coesão pode ser dividida em coesão de tarefa e coesão social. A coesão de tarefa encontra-se quando o grupo está coeso em volta de uma tarefa, e organizado para desempenhá-la, como por exemplo, ganhar um campeonato. Em contrapartida, a coesão social pode ser entendida quando o grupo está coeso em volta de funções sociais, refletindo a atração interpessoal entre os membros do grupo, como por exemplo, o atleta valoriza mais as amizades da equipe do que as vitórias da equipe. (XAVIER et al., 2020).

A respeito dos instrumentos existentes para avaliações no contexto esportivo, pode-se citar o Group Environment Questionaire (GEQ), desenvolvido por Carron, Widmeyer e Brawley (1985), adaptado e validado para o Brasil por Nascimento Junior et al. (2012), intitulado, no Brasil, de Questionário de Ambiente de Grupo (GEQ). Esse instrumento apresenta boa consistência interna, demonstrando validade psicométrica para seu uso. (NASCIMENTO JUNIOR et al., 2012, 2016b; TERTULIANO et al., 2019; XAVIER et al., 2020).

Utilizando-se desses instrumentos, alguns estudos foram conduzidos, com o intuito de avaliar os níveis de coesão de grupo no futebol e futsal (FIORESE et al., 2017; NASCIMENTO JUNIOR et al., 2011, 2016b; TERTULIANO et al., 2019), no voleibol (BALBIM; NASCIMENTO JUNIOR; VIEIRA, 2012; NASCIMENTO JUNIOR; BALBIM; VIEIRA, 2013), no handebol (ALVES, 2010; NASCIMENTO JUNIOR et al., 2016a), no basquetebol (ALVES, 2010; XAVIER et al., 2020) e em outros esportes (LEITE et al., 2015; SILVEIRA; OLIVEIRA, 2017). Além disso, a maioria dos estudos foram realizados com atletas com 
idade superior a 20 anos (BALBIM; NASCIMENTO JUNIOR; VIEIRA, 2012; FIORESE et al., 2017; LEITE et al., 2015; NASCIMENTO JUNIOR et al., 2011, 2016a, 2016b; NASCIMENTO JÚNIOR et al., 2018; NASCIMENTO JUNIOR; BALBIM; VIEIRA, 2013; SILVEIRA; OLIVEIRA, 2017), demonstrando a necessidade de estudos com atletas mais jovens, pois a literatura cita que a idade pode ser um fator que influencia os níveis de coesão da equipe. (NASCIMENTO JUNIOR; VIEIRA, 2013; TERTULIANO et al., 2019).

Tertuliano et al. (2019) investigaram a coesão de grupo em atletas de futebol com idade entre 15 e 19 anos, e observaram que os atletas mais velhos apresentam maior coesão relacionado a tarefa, quando comparados aos atletas mais jovens, justificando o presente estudo. Ainda, em relação a modalidade esportiva basquetebol, modalidade investigada no presente estudo, apenas um artigo foi conduzido. (XAVIER et al., 2020) exclusivamente com atletas de basquetebol, tendo o objetivo de investigar os níveis de coesão de grupo em atletas de basquetebol com idade entre 13 e 15 anos (o mesmo público-alvo do presente manuscrito). Os autores avaliaram os atletas em dois momentos, início e final da competição.

Nesse estudo, os resultados demonstraram que os atletas apresentaram maiores escores para dimensão atração individual para o grupo-tarefa (AI-T), nos dois momentos da competição, e que não houve mudança de escores na comparação entre o início e o final da competição. (XAVIER et al., 2020). Todavia, cabe apontar que esse foi o único estudo com essa modalidade e com essa faixa etária, no mesmo estudo, o que demonstra escassez de estudos e, assim, justifica o presente estudo.

O estudo de Alves (2010) investigou atletas de basquetebol e handebol, pois considerou no estudo atletas de esportes coletivos. Entretanto, o basquetebol apresenta algumas características peculiares que podem influenciar nos níveis de coesão, como o fato do ataque ter a obrigatoriedade de concluir um ataque em 24 segundos, regra que só existe no basquetebol e que pode ter influência sobre diferentes condições psicológicas da equipe, inclusive a coesão de grupo, justificando o presente estudo. Além disso, na concepção do esporte, atletas amadores se comportam diferentemente de atletas profissionais (MACHADO, 2006), pois as cobranças e exigências são distintas.

Diante disso, boa parte dos estudos supracitados foram conduzidos com atletas profissionais e adultos, o que demonstra a necessidade de estudos com atletas amadores (adolescentes), justificando, novamente, o presente estudo. Cabe citar, também, que os níveis de coesão dos atletas podem modificar durante o campeonato (CARRON; WIDMEYER; BRAWLEY, 1985; WEINBERG; GOULD, 2017; XAVIER et al., 2020), o que foi avaliado apenas em um artigo apontado acima (XAVIER et al., 2020), reforçando a justificativa do presente estudo. Portanto, o presente estudo teve o objetivo de investigar a relação entre a idade (sub-13 e sub-15) e o momento da competição (início e final do campeonato) com os níveis de coesão de grupo em atletas amadores de basquetebol. 


\section{METODOLOGIA}

\subsection{PARTICIPANTES}

A pesquisa contou com a participação voluntária de 26 atletas amadores das categorias de base de um clube de uma cidade da região metropolitana do estado de São Paulo, todos atletas de basquetebol, com idade entre 13 e 15 anos, federados e do sexo masculino. Os participantes pertencem a 2 categorias do Basquetebol, a sub13 (12 participantes) e a sub-15 (14 participantes). Para atender ao objetivo do estudo, os atletas foram divididos em 2 grupos amostrais, sendo: Grupo 1 - Atletas da categoria sub-13 (12 participantes), com média de idade de 13,75 anos e DP de 0,42 anos; e, Grupo 2 - Atletas da categoria sub-15 (14 participantes), com média de idade de 14,57 anos e DP de 0,51 anos.

No presente estudo, com o intuito de controlar algumas variáveis que poderiam influenciar os resultados, a amostra foi formada por conveniência. Assim, alguns critérios foram adotados, como a seleção de atletas que eram treinados pela mesma equipe técnica (treinador e auxiliares), pertencentes ao mesmo clube e que disputaram o mesmo campeonato. Dessa forma, a presente pesquisa se constitui como um estudo de caso. (THOMAS; NELSON; SILVERMAN, 2012).

\subsection{INSTRUMENTO}

Os participantes responderam ao Questionário de Ambiente de Grupo - GEQ (CARRON; WIDMEYER; BRAWLEY, 1985), adaptado e validado para o Brasil por Nascimento Junior et al. (2012). Este instrumento é composto por 16 itens que avaliam a coesão de grupo em equipes esportivas.

Os itens são distribuídos em quatro dimensões: 1- Integração no Grupo-Tarefa (GI-T) como o atleta considera a harmonia da equipe em tarefas a serem realizadas (itens 8 , 10, 12, 14 e 16); 2- Integração no Grupo-Social (GI-S) - como o atleta considera a união da equipe como unidade social (itens 9, 11, 13 e 15); 3- Atração Individual para o Grupo-Tarefa (Al-T) - como o atleta considera seu envolvimento com os objetivos estabelecidos (itens 3, 4 e 6); e, 4- Atração Individual para o Grupo-Social (Al-S) como o atleta considera a sua integração dentro do grupo social (itens 1, 2, 5 e 7).

O instrumento é respondido em uma escala do tipo likert de 9 pontos. As respostas são colocadas de maneira que o avaliando escolha uma opção de um a nove para cada resposta, partindo do pressuposto que 1 corresponde a "discordo totalmente" e 9 "concordo totalmente". Nesse instrumento, as respostas mais próximas de 9 "concordo totalmente", indicam um alto nível de coesão, enquanto que, as respostas mais próximas de 1 "discordo totalmente", indicam um nível menor de coesão. Para calcular o escore de cada dimensão deve-se somar as respostas dos itens de cada dimensão e dividir pelo respectivo número de itens, podendo variar, o valor, entre 1 e 9 . 


\subsection{PROCEDIMENTOS}

O presente estudo seguiu os princípios éticos de uma pesquisa com seres humanos, ou seja, partindo de etapas estabelecidas pelo meio científico e respeitando todos os padrões éticos de pesquisas com seres humanos do Conselho Nacional de Saúde (CNS), Resolução 466/12. O presente trabalho foi apresentado ao Comitê de Ética e Pesquisa (CEP) do Hospital e Centro de Reabilitação da AACD e aprovado sob o número de parecer: 1.541.273.

Posteriormente a autorização do CEP, contatou-se o clube para autorização da coleta com seus respectivos atletas. Após a autorização do clube e dos técnicos, os atletas foram informados dos objetivos do estudo e assinaram um termo de consentimento livre e esclarecido (TCLE), garantindo-Ihes o anonimato e confiabilidade de todas as informações coletadas. Como todos atletas são menores de idade, os mesmos assinaram o termo de assentimento e um responsável legal, maior de idade, assinou o TCLE.

Respeitando o objetivo do estudo, alguns cuidados metodológicos foram adotados para garantir a confiabilidade das informações coletas, de acordo com as previsões teóricas acerca da coesão de grupo. Para tanto, os atletas foram orientados a responder o questionário individualmente em uma sala no clube, junto da presença de um dos pesquisadores. Tais medidas adotadas ocorreram para evitar o contato entre os atletas e entre o atleta e a comissão técnica durante o preenchimento do questionário. Assim, não houve a troca de informações pelos jogadores antes e durante a aplicação do questionário.

Além disso, os atletas responderam ao questionário em 2 momentos: no início do campeonato (na semana da primeira partida no torneio, para os dois grupos, que ocorreu em abril de 2018) e no final do campeonato (na semana do jogo final das equipes, que coincidiu com as finais do torneio - para as duas equipes, que ocorreu em novembro de 2018). O tempo de preenchimento do questionário foi de aproximadamente 15 minutos. Após os atletas responderem o questionário, as informações coletadas foram transferidas para uma planilha eletrônica (Excel, versão 2016), para análise dos resultados.

\subsection{ANÁLISE ESTATÍSTICA}

No presente estudo as análises inferenciais assumiram o valor de $p \leq 0,05$ para níveis de significância. Em função do número de participantes ( $n<30$ participantes), se assumiu a ausência de normalidade, apoiando-se no teorema do limite central (GREEN; SALKIND; AKEY, 2000; PAGANO; GAUVREAU, 2004; TRIOLA, 2017), e adotouse testes não paramétricos. Dessa forma, foram utilizados mediana (Md) e quartis (Q1; Q3) para caracterização dos resultados.

Com o intuito de avaliar a confiabilidade das dimensões do instrumento, ou seja, avaliar a consistência interna das dimensões do questionário, em cada momento de coleta, utilizou-se do alfa de Cronbach ( $\alpha$ de Cronbach), assumindo valores entre 0,7 e 0,8 como aceitáveis para o $\alpha$ (FIELD, 2009), para cada dimensão e momento, de forma isolada. Para avaliação da fidedignidade teste-reteste foi utilizado o coeficiente de 
correlação intraclasses entre os itens e dimensões do instrumento. Os valores de correlação foram avaliados conforme proposto por Hopkins (2002), em que <0,10 (trivial), 0,10 a 0,30 (baixa), 0,31 a 0,50 (moderada), 0,51 a 0,70 (alta), 0,71 a 0,90 (muito alta), 0,91 a 0,99 (quase perfeita) e 1 (perfeita).

Para analisar as diferenças entre os grupos (no início e no final do campeonato), utilizou-se o teste $U$ de Mann-Whitney. No que tange a compreensão dos níveis de coesão de grupo (análise intragrupo), utilizou-se o teste de Friedman. Concernente à localização, utilizou-se o post hoc de Wilcoxon. Para controle do erro tipo 1, foi utilizado o procedimento sequencial Holm de Bonferroni. (GREEN; SALKIND; AKEY, 2000). Todas as análises foram realizadas com o auxílio do IBM SPSS Statistics, versão 22.

Em relação à confiabilidade das escalas, o alpha de Cronbach revelou bons índices de confiabilidade no início $(\alpha>0,868)$ e no final do campeonato $(\alpha>0,572)$, ou seja, o índice de consistência interna geral do instrumento foi satisfatório nos dois momentos de coleta. Entretanto, como o instrumento é multidimensional, houve a necessidade de calcular a confiabilidade de cada dimensão, em cada momento de coleta, isoladamente.

Os valores de confiabilidade para cada dimensão, no início do campeonato, variaram entre 0,868 e 0,929, tendo a correlação item-dimensão variando entre 0,569 e 0,864, indicando correlação alta entre os itens e a dimensão a qual ele pertence. No que tange aos resultados para o final do campeonato, os valores de confiabilidade para cada dimensão variaram entre 0,572 e 0,884 , tendo a correlação item-dimensão variando entre 0,514 e 0,837, indicando correlação alta entre os itens e a dimensão a qual eles pertencem.

\section{RESULTADOS}

Nas comparações entre grupos conduziu-se análises para cada momento da coleta. Assim, analisando-se o início do campeonato, pode-se observar que o grupo Sub-13 apresentou valores superiores para quase todas as dimensões (Tabela 1). Tais informações não foram confirmadas pelas análises inferenciais, conduzidas pelo teste $U$ de Mann-Whitney, pois o mesmo não apresentou diferença significante entre os grupos, para nenhuma das dimensões do instrumento $(p>0,05)$. Tais informações apontam que os dois grupos apresentaram níveis de coesão de grupo similares no início do campeonato.

Analisando-se o final do campeonato, pode-se observar que o grupo Sub-15 apresentou valores superiores para quase todas as dimensões (Tabela 2). Tais informações, novamente, não foram confirmadas pelas análises inferenciais, pois o teste não apresentou diferença significante entre os grupos, para nenhuma das dimensões do instrumento $(p>0,05)$. Tais informações apontam, estatisticamente, que os dois grupos apresentaram níveis de coesão de grupo similares no final do campeonato. 
Tabela 1 - Comparação das dimensões de coesão de grupo no início do campeonato $(n=26)$.

\begin{tabular}{|c|c|c|c|c|c|}
\hline \multirow{2}{*}{\multicolumn{2}{|c|}{ Dimensão }} & \multirow{2}{*}{$\begin{array}{l}\text { Sub } 13(n=12) \\
\text { Md (Q1; Q3) }\end{array}$} & \multirow{2}{*}{$\begin{array}{l}\text { Sub } 15(n=14) \\
\text { Md (Q1; Q3) }\end{array}$} & \multirow{2}{*}{$\begin{array}{l}\text { Mann- } \\
\text { Whitney } \\
\text { U }\end{array}$} & \multirow{2}{*}{$\mathbf{P}$} \\
\hline & & & & & \\
\hline \multicolumn{2}{|c|}{ 1. Integração no Grupo-Tarefa (GI-T) } & $7,50(7,25 ; 7,90)$ & $7,00(6,35 ; 7,65)$ & 48,000 & 0,067 \\
\hline \multicolumn{2}{|c|}{ 2. Integração no Grupo-Social (GI-S) } & $6,75(4,94 ; 7,25)$ & $6,00(5,13 ; 7,32)$ & 75,000 & 0,667 \\
\hline \multicolumn{2}{|c|}{$\begin{array}{l}\text { 3. Atração Individual para o Grupo-Tarefa } \\
\text { (Al-T) }\end{array}$} & $8,00(7,09 ; 8,67)$ & $7,83(6,25 ; 8,42)$ & 71,500 & 0,527 \\
\hline \multicolumn{2}{|c|}{$\begin{array}{l}\text { 4. Atração Individual para o Grupo-Social } \\
\text { (AI-S) }\end{array}$} & $7,12(6,38 ; 7,94)$ & $7,50(6,07 ; 8,07)$ & 75,500 & 0,667 \\
\hline \multirow{2}{*}{ Friedman } & $\mathbf{X}^{2}$ & 19,552 & 17,773 & & \\
\hline & $\mathbf{P}$ & $0,001 *$ & $0,001 *$ & & \\
\hline
\end{tabular}

Fonte: Elaborada pelos autores. *diferença significante.

Tabela 2 - Comparação das dimensões de coesão de grupo no final do campeonato $(n=26)$.

\begin{tabular}{|c|c|c|c|c|c|}
\hline \multirow{2}{*}{\multicolumn{2}{|c|}{ Dimensão }} & Sub $13(n=12)$ & Sub $15(n=14)$ & \multirow{2}{*}{$\begin{array}{l}\text { Mann- } \\
\text { Whitney } \\
\text { U }\end{array}$} & \multirow{2}{*}{$\mathbf{P}$} \\
\hline & & Md (Q1; Q3) & Md (Q1; Q3) & & \\
\hline \multicolumn{2}{|c|}{ 1. Integração no Grupo-Tarefa (GI-T) } & $7,60(7,05 ; 8,40)$ & $7,10(5,60 ; 7,65)$ & 53,000 & 0,110 \\
\hline \multicolumn{2}{|c|}{ 2. Integração no Grupo-Social (GI-S) } & $5,38(4,06 ; 6,68)$ & $5,62(5,00 ; 6,87)$ & 70,000 & 0,470 \\
\hline \multicolumn{2}{|c|}{$\begin{array}{l}\text { 3. Atração Individual para o Grupo- } \\
\text { Tarefa (Al-T) }\end{array}$} & $8,17(6,67 ; 8,67)$ & $8,33(7,50 ; 8,67)$ & 80,500 & 0,855 \\
\hline \multicolumn{2}{|c|}{$\begin{array}{l}\text { 4. Atração Individual para o Grupo- } \\
\text { Social (AI-S) }\end{array}$} & $7,00(5,81 ; 8,13)$ & $7,12(5,94 ; 8,00)$ & 83,000 & 0,959 \\
\hline \multirow{2}{*}{ Friedman } & $\mathbf{x}^{2}$ & 26,128 & & & \\
\hline & $\mathbf{P}$ & $0,001 *$ & $0,001 *$ & & \\
\hline
\end{tabular}

Fonte: Elaborada pelos autores.

*diferença significante.

Com a intenção de verificar se os grupos apresentaram elevados níveis de coesão, pode-se observar que os dois grupos apresentaram, tanto no início, quanto no final da competição, escores superiores para dimensão Al-T e elevados níveis de coesão geral, pois apresentaram escores superior a cinco (NASCIMENTO JUNIOR et al., 2012; TERTULIANO et al., 2019) para todas as dimensões (Tabelas 1 e 2).

Referente ao nível de coesão do sub-13, o teste de Friedman demonstrou diferenças significantes entre as dimensões no início do campeonato $\left[x^{2}(3)=19,552 ; p<0,001\right]$ e no final do campeonato $\left[x^{2}(3)=26,168 ; p<0,001\right]$. Concernente a localização, o post hoc apontou que no início do campeonato houve diferença significante apenas entre a dimensão Al-T e GI-S, demonstrado que a subescala GI-S obteve os menores escores no início do campeonato. Em relação ao final do campeonato, o post hoc apresentou as mesmas diferenças apresentadas no início do campeonato, ou seja, a dimensão Al-T obteve escores superiores apenas em relação à dimensão $\mathrm{Gl}-\mathrm{S}(p<0,008$, valor ajustado). 
No que se refere o nível de coesão do sub-15, o teste de Friedman demonstrou diferenças significantes entre as dimensões no início do campeonato $\left[x^{2}(3)=17,773\right.$; $p<0,001]$ e no final do campeonato $\left[x^{2}(3)=26,128 ; p<0,001\right]$. Concernente à localização, o post hoc apontou que no início do campeonato houve diferença significante entre a dimensão AI-T e a dimensão GI-S ( $p<0,008$, valor ajustado), a qual obteve o menor escore do início do campeonato. Em relação ao final do campeonato, o post hoc demonstrou que a dimensão Al-T obteve escores superiores as demais dimensões ( $p<0,008$, valor ajustado).

Em síntese, os resultados deste estudo demonstram que independentemente do momento da coleta (início ou final do campeonato), não houve diferenças significantes entre os grupos, e que ambos apresentaram bons níveis de coesão de grupo, com a dimensão Al-T apresentando os maiores escores para os dois grupos, nos dois momentos da competição.

\section{DISCUSSÕES}

O presente estudo teve o objetivo de investigar a relação entre a idade (sub-13 e sub15) e o momento da competição (início e final do campeonato) com os níveis de coesão de grupo em atletas amadores de Basquetebol. Em relação aos resultados do presente estudo, os achados demonstraram que os dois grupos apresentaram resultados similares, ou seja, não houve diferenças significantes entre eles. Tais resultados não corroboram a literatura, pois ela cita que a faixa etária parece ser determinante no nível de coesão. (TERTULIANO et al., 2019). Tertuliano et al. (2019) avaliaram os níveis de coesão em equipes de futebol. Participaram do estudo adolescentes com idade entre 13 e 19 anos. Os resultados demonstraram que, apesar de todos os grupos etários avaliados apresentarem maiores escores para dimensão atração individual no grupo-tarefa (AI-T), o grupo com atletas mais velhos foi superior nas duas dimensões relacionadas a coesão pela tarefa. Os autores concluíram que os atletas mais velhos do estudo, próximo da vida adulta (entre 17 e 19 anos), podem estar mais envolvidos com a coesão pela tarefa, pois apresentam a meta de se profissionalizar e nesse momento da vida as amizades não influenciam de forma significante a coesão em prol dos bons resultados.

Tais apontamentos remetem ao entendimento de que atletas mais velhos atuam para alcançar objetivos comuns, como por exemplo conquistar um campeonato (WEINBERG; GOULD, 2017), e se dedicar mais aos treinamentos, além de envolver mais com a equipe (APPLE, 1993), corroborando a literatura que investigou a temática em atletas mais velhos (CARRON; WIDMEYER; BRAWLEY, 1985; ESTABROOKS; CARRON, 2000; EYS et al., 2009; FIORESE et al., 2017; NASCIMENTO JUNIOR et al., 2012, 2016a, 2016b). Nascimento Júnior e Vieira (2013) avaliaram os níveis de coesão em equipes de futsal e verificaram que a coesão para tarefa é mais relevante para atletas mais velhos (comparação entre idades - $25 \times 26$ anos de idade), quando comparada com a coesão social. Entretanto, é relevante apontar que o estudo de Nascimento Júnior e Vieira (2013) se utilizou de adultos e o presente estudo de adolescentes, o que pode ter influenciado a diferença entre os resultados dos estudos, ou seja, enquanto o presente estudo não encontrou diferenças entre os grupos, o estudo de Nascimento Júnior e Vieira (2013) encontrou. Além disso, no estudo de 
Tertuliano et al. (2019) utilizou-se atletas de futebol, o que também pode ter relação com a diferença entre os resultados do presente estudo e os apresentados pelos autores.

Dessa forma, investigando os níveis de coesão em atletas jovens e da modalidade basquetebol, Xavier et al. (2020) investigaram os níveis de coesão de grupo de atletas de base do basquetebol com idade entre 13 e 15 anos, comparando os níveis de coesão no início e no final de uma competição. Os resultados demonstraram que os atletas apresentaram níveis mais elevados para dimensão Al-T (Atração Individual para o Grupo-Tarefa), tanto no início, quanto no final da competição, além de não demonstrarem diferenças entre o início e o final da competição. Os autores assumiram que tais resultados são favoráveis para o desempenho esportivo e que estavam associados a satisfação dos atletas.

Alves (2010) investigou os níveis de coesão de grupo e sua relação com o sexo dos atletas, a modalidade (handebol e basquetebol), o clima motivacional e a satisfação dos atletas. Participaram do estudo 979 atletas, de ambos os sexos, com idade entre 11 e 16 anos. Os resultados demonstraram que para o basquetebol, os resultados de coesão de grupo foram mais significativos para duas dimensões atração individual para o grupo-tarefa (Al-T) e atração individual para o grupo-social (Al-S), e que esses resultados tem relação com o clima motivacional orientado para tarefa e com a satisfação dos atletas com a equipe.

Tais apontamentos parecem ser bem coerentes para explicar os achados do presente estudo, pois os dois grupos apresentaram elevados escores de coesão de grupo para dimensão Al-T e para coesão geral (todas dimensões com escores acima de cinco) (CARRON; WIDMEYER; BRAWLEY, 1985; NASCIMENTO JUNIOR et al., 2012; TERTULIANO et al., 2019), tanto no início, quanto no final da competição. Esses resultados podem sugerir que quanto maior forem os escores relacionados a tarefa, maior será o empenho dos atletas para atingir os objetivos estabelecidos pela equipe, corroborando os achados de Alves (2010) e de outros estudos da literatura. (EYS et al., 2009; TERTULIANO et al., 2019; XAVIER et al., 2020). Além disso, parece que para os atletas adolescentes de basquetebol, a coesão tende a apresentar maiores escores para dimensão Al-T.

A literatura cita que níveis elevados de Al-T são importantes para equipe, pois demonstram que os atletas estão focados nos objetivos de vitória e conquistas, independentemente de seus companheiros de equipe serem amigos ou apenas colegas de equipe (BRISIMIS; BEBETSOS; KROMMIDAS, 2018; CARRON; WIDMEYER; BRAWLEY, 1985; ERIKSTAD et al., 2018; ESTABROOKS; CARRON, 2000; EYS et al., 2007; FIORESE et al., 2017; MORÃO et al., 2019; NASCIMENTO JÚNIOR et al., 2018; NASCIMENTO JUNIOR; VIEIRA, 2013; SEZER; KOCAEKSI, 2018; TERTULIANO et al., 2019; VERZANI et al., 2019; XAVIER et al., 2020). Em síntese, os elevados escores de Al-T, podem contribuir para o que o atleta obtenha sucesso esportivo (CARRON; BRAY; EYS, 2002) e se demonstre mais envolvido com a equipe. (XAVIER et al., 2020).

Assumindo a relação entre satisfação e coesão, apontadas por Alves (2010), os resultados do presente estudo podem sugerir que os atletas deste estudo, possivelmente, tinham satisfação em participar da equipe, pois demonstraram grande envolvimento com o objetivo da equipe (WEINBERG; GOULD, 2017), reforçando o 
supracitado e corroborando outros estudos. (FIORESE et al., 2017; NASCIMENTO JÚNIOR et al., 2018; TERTULIANO et al., 2019).

O nível competitivo também é apontado como uma variável que influencia os níveis de coesão do grupo. (BALBIM; NASCIMENTO JUNIOR; VIEIRA, 2012; NASCIMENTO JUNIOR et al., 2016a; NASCIMENTO JUNIOR; VIEIRA, 2013). Nascimento Junior e Vieira (2013) compararam os escores de equipes que disputavam campeonatos de diferentes níveis competitivos (Estadual e Nacional). Os autores observaram que quanto maior é o nível competitivo da competição, maior são os escores de coesão apresentados pelas equipes. Por se tratar de uma equipe federada, a coesão relacionada à tarefa se torna mais importante do que a coesão social, pois as equipes federadas apresentam maior cobrança em vários aspectos, tanto por parte do atleta, como por parte da equipe.

Em resumo, a literatura aponta que atletas profissionais apresentam maior coesão de grupo em prol da tarefa, e que atletas amadores apresentam maiores escores em prol do convívio social (NASCIMENTO JUNIOR; VIEIRA, 2013), o que não foi corroborado pelo presente estudo, pois os resultados demonstraram que as duas equipes investigadas (sub-13 e sub-15, ambas amadoras) obtiveram maiores escores para coesão em prol da tarefa. Entretanto, é oportuno citar que os estudos que apontaram a influência do nível competitivo utilizaram-se de adultos (BALBIM; NASCIMENTO JUNIOR; VIEIRA, 2012; NASCIMENTO JUNIOR et al., 2016a; NASCIMENTO JUNIOR; VIEIRA, 2013), não de adolescentes, limitando a discussão acerca dessa variável junto aos achados do presente estudo.

Morão et al. (2019) investigaram os níveis de coesão em 162 atletas de futebol com média de idade de 17,95 anos, idade próxima a dos atletas investigados no presente estudo. Os resultados demonstraram que a posição em que os jogadores atuam influenciou os níveis de coesão para o envolvimento em prol da tarefa e do convívio social. Esses resultados sugerem que outros fatores podem influenciar os níveis de coesão de grupo, como por exemplo, o estilo de liderança do treinador (NASCIMENTO JUNIOR; VIEIRA, 2012), os estados de humor dos atletas (VIEIRA et al., 2008), os níveis de ansiedade dos atletas (NASCIMENTO JUNIOR et al., 2016a; PAES et al., 2016) e a posição de jogo dos atletas (MORÃO et al., 2019), os quais não foram objetivo do presente estudo, mas podem auxiliar nas hipóteses explicativas dos achados deste manuscrito.

Tratando-se do estilo de liderança do treinador, as equipes treinadas por profissionais de perfis mais democráticos apresentaram maior coesão de grupo (NASCIMENTO JUNIOR; VIEIRA, 2012), tanto para dimensão da tarefa quanto para dimensão social. Além disso, atletas mais jovens tendem a avaliar a liderança de forma mais favorável em relação ao reforço positivo, quando comparados a atletas mais velhos (GOMES; PEREIRA; PINHEIRO, 2008), o que pode influenciar a percepção do atleta sobre o papel da liderança na coesão do grupo.

Assumindo esses apontamentos para explicar os achados do presente estudo, eles podem justificar os resultados, pois os atletas do presente estudo eram treinados pela mesma equipe técnica (sub-13 e sub-15), demonstrando que a ausência de diferenças entre os dois grupos avaliados pode, em tese, ter relação com o estilo de liderança, haja vista as duas equipes terem recebido o mesmo tipo de orientação e intervenção 
técnica. Todavia, essas hipóteses explicativas não foram objetivo do presente estudo, servindo apenas para direcionar possíveis influências do técnico nos resultados (WERNECK et al., 2015) e perspectivas futuras de estudos.

Em relação a influência da ansiedade na coesão de grupo, Paes et al. (2016) investigaram a correlação entre coesão de grupo e ansiedade pré-competitiva de atletas de voleibol durante uma competição. Os autores avaliaram 12 atletas do sexo masculino com média de idade de 16 anos. Os resultados demonstraram que os atletas apresentaram maiores escores para três dimensões: Al-T (atração individual para o grupo-tarefa), Al-S (atração individual para o grupo-social) e GI-T (integração no grupo-tarefa). Além disso, os atletas demonstraram elevados escores de autoconfiança e baixos escores de ansiedade somática e cognitiva. Em relação a correlação entre ansiedade e coesão, os resultados demonstraram que houve correlação positiva entre a autoconfiança e a coesão social (Al-S e GI-S - integração no grupo-social). Os autores assumiram que essa correlação foi importante para os atletas se sentirem mais motivados e satisfeitos com o grupo e, assim, dedicar-se mais com as metas do grupo, o que conduziu aos elevados escores de coesão pela tarefa (Al-T e GI-T).

A literatura cita, também, que além da ansiedade, o sexo dos atletas pode influenciar os níveis de coesão durante as competições. (NASCIMENTO JUNIOR et al., 2016a). Nascimento Junior et al. (2016a) investigaram a influência dos níveis de ansiedade e do sexo dos atletas nos níveis de coesão durante uma competição. Os autores investigaram 62 atletas de ambos os sexos, com média de idade de 23 anos. Os resultados apontaram que para os homens, os níveis de coesão pela tarefa aumentaram durante a competição, enquanto que para as mulheres diminuíram. Referente a ansiedade, os resultados demonstraram que as mulheres reduziram os níveis de ansiedade somática durante a competição, e que os homens não apresentaram alterações nos níveis de ansiedade. Pode-se observar, também, que as mulheres apresentaram maior autoconfiança no final da competição, comparadas aos homens, mas que os homens apresentaram maior coesão pela tarefa no jogo final.

Em relação a correlação, os resultados evidenciaram que para as mulheres, a correlação foi entre a ansiedade somática e as dimensões: Al-T (atração individual para o grupo-tarefa), GI-T (integração no grupo-tarefa) e GI-S (integração no gruposocial). Para os homens, houve correção entre autoconfiança e as dimensões: Al-T, GI-T e Al-S (atração individual para o grupo-social). Além disso, durante a competição os homens apresentaram maiores escores para coesão pela tarefa, enquanto as mulheres tiveram maiores escores para coesão social. Os autores concluíram que homens e mulheres, no que tange a correlação entre ansiedade e coesão, apresentam resultados distintos, sendo que para o homem a autoconfiança influencia de forma significativa a coesão pela tarefa, ou seja, quanto menor for a autoconfiança, mais elevados são os escores de coesão pela tarefa. Para as mulheres, é a ansiedade somática que influencia os níveis de coesão, ou seja, quanto mais elevada for a ansiedade somática, maiores são os escores de coesão social. (NASCIMENTO JUNIOR et al., 2016a).

Todavia, como já citado, essas hipóteses explicativas são apresentadas como possíveis explicações dos resultados encontrados no presente estudo, mas não são possíveis de 
serem suportadas pelos resultados do presente estudo, pois tais relações não foram objetivo do presente estudo, apontando, assim, perspectivas futuras de estudo. Por fim, o presente estudo apresenta algumas limitações, como ter investigado atletas de um único clube (estudo de caso), ter como participantes apenas atletas do sexo masculino e de uma única modalidade esportiva, e não ter relacionado a coesão de grupo com outras variáveis, como ansiedade, por exemplo. Entretanto, cabe apontar que no presente estudo as limitações foram assumidas desde o início, pois optou-se por selecionar participantes por conveniência e avaliar os níveis de coesão da equipe em diferentes momentos da competição. Em outras palavras, essas limitações não são consideradas limitações metodológicas, mas limitações para generalização dos resultados.

\section{CONSIDERAÇÕES FINAIS}

O presente estudo objetivou investigar a relação entre a idade (sub-13 e sub-15) e o momento da competição (início e final do campeonato) com os níveis de coesão de grupo em atletas amadores de Basquetebol. Os resultados demonstraram que a idade e o momento da competição não influenciaram os resultados, pois os grupos (sub-13 $x$ sub-15) não demonstraram diferença significante, independentemente do período da competição (início ou final da competição). No que se refere o nível de coesão dos grupos, os dois grupos apresentaram bons níveis de coesão, com a dimensão Al-T (atração individual para o grupo-tarefa) apresentando os maiores escores, para ambos, independentemente do momento da competição.

Enquanto implicações práticas, os resultados do presente estudo, bem como outros achados da literatura (ALVES, 2010; PAES et al., 2016; TERTULIANO et al., 2019; XAVIER et al., 2020), sugerem que a coesão de grupo deve ser um fator importante para os técnicos observarem nas categorias de base, pois esse é um fator relevante para o desempenho da equipe. Sendo assim, pode-se dizer que a formação de um grupo vitorioso tem grande influência da coesão que o grupo demonstra, sendo que a coesão pela tarefa parece ser mais relevante para o sucesso esportivo, principalmente no basquetebol.

Finalizando, sugere-se que para estudos futuros, a amostra seja constituída por atletas de ambos os sexos, abordando um maior número de modalidades e em maior escala territorial (mais de um clube), pois dessa forma é possível obter uma melhor análise da coesão de grupo em diferentes contextos e públicos e, assim, generalizar os achados. Por fim, espera-se, também, que estudos futuros relacionem coesão de grupo com outras variáveis, como por exemplo liderança, estado de humor e ansiedade.

\section{REFERÊNCIAS}

ALMEIDA, P. H. G. L. Variables psicológicas y rendimiento deportivo en el fútbol profesional. 2008. 378 f. Tese (Doutorado em Psicologia) - Universidad Nacional de Educación a Distancia, Madrid, 2008. 
ALVES, I. Relação entre a coesão, o clima motivacional e a satisfação em modalidades colectivas. 2010. 208 f. Dissertação (Mestrado em Psicologia do Desporto e do Exercício) - Escola Superior de Desporto de Rio Maior, Rio Maior, 2010.

APPLE, $K$. The antecedents and consequences of multidimensional cohesion throughout an intercollegiate baseball season. 1993. 254 f. Thesis (Doctorate in Psychology) - Purdue University, Indiana, 1993.

ASAMOAH, B.; GROBBELAAR, H. W. Team cohesion and performance during a university soccer championship: two sides of the coin. South African Journal for Research in Sport, Physical Education and Recreation, Cidade do Cabo, v.39, n.1, p.17-31, 2017.

BALBIM, G. M.; NASCIMENTO JUNIOR, J. R. A.; VIEIRA, L. F. Análise do nível de coesão de grupo e do estresse psicológico pré-competitivo de atletas adultos de voleibol.

Revista Brasileira de Cineantropometria \& Desempenho Humano, Florianópolis, v.14, n.6, p.704-712, 2012.

BRISIMIS, E.; BEBETSOS, E.; KROMMIDAS, C. Does group cohesion predict team sport athletes' satisfaction? Hellenic Journal of Psychology, Thessaloniki, v.15, n.1, p.108-124, 2018.

CARRON, A. V.; WIDMEYER, W. N.; BRAWLEY, L. The development of an instrument to assess cohesion in sport teams: the group environment questionnaire. Journal of Sport Psychology, London, v.7, n.3, p.244-266, 1985.

CARRON, A. V; BRAY, S. R.; EYS, M. A. Team cohesion and team success in sport. Journal of Sport Sciences, London, v.20, p.119-226, 2002.

ERIKSTAD, M. K.; et al. Group cohesion, needs satisfaction, and self-regulated learning: A one-year prospective study of elite youth soccer players' perceptions of their club team. Psychology of Sport and Exercise, London, v.39, p.171-178, 2018.

ESTABROOKS, P. A.; CARRON, A. V. The physical activity group environment questionnaire: an instrument for the assessment of cohesion in exercise classes.

Group dynamics: theory, research, and practice, Washington, v.4, n.3, p.230-243, 2000.

EYS, M. A. et al. Item wording and internal consistency of a measure of cohesion: the group environment questionnaire. Journal of sport \& exercise psychology, Birmingham, v.29, p.395-402, 2007.

EYS, M. A. et al. Development of a cohesion questionnaire for youth: the Youth Sport Environment Questionnaire. Journal of sport \& exercise psychology, Bethesda, v.31, n.3, p.390-408, 2009.

FIELD, A. Descobrindo a estatística usando o SPSS. São Paulo: Bookman, 2009.

FIORESE, L. et al. Associação entre motivação e coesão de grupo no futebol profissional: o relacionamento treinador-atleta é um fator determinante? Revista de Psicología del Deporte, Barcelona, v.27, n.suppl.1, p.51-57, 2017. 
GOMES, A. R.; PEREIRA, A. P.; PINHEIRO, A. R. Liderança, coesão e satisfação em equipas desportivas: um estudo com atletas Portugueses de futebol e futsal.

Psicologia: Reflexão e Crítica, Porto Alegre, v.21, n.3, p.482-491, 2008.

GREEN, S. B.; SALKIND, N. J.; AKEY, T. M. Using SPSS for Windows: analyzing and understanding data. 2. ed. New Jersey: Prentice Hall, 2000.

LEITE, M. A. F. J. et al. Nível de coesão grupal e satisfação de atletas de rugby. Coleção Pesquisa em Educação Física, Várzea Paulista, v.14, n.3, p.125-132, 2015.

MACHADO, A. A. Psicologia do esporte: da educação física escolar ao esporte de alto nível. Rio de Janeiro: Guanabara Koogan, 2006.

MIKALACHKI, A. Group cohesion reconsidered. London, Ontario: University of Western Ontario, 1969.

MORÃO, K. G. et al. Estudo exploratório da Coesão em atletas juniores de futebol.

Educación Física y Ciencia, Buenos Aires, v.21, n.2, p.e082, 2019.

NASCIMENTO JUNIOR, J. R. A.; BALBIM, G. M.; VIEIRA, L. F. Coesão de grupo em equipes adultas de voleibol do estado do Paraná. Revista Psicologia: Teoria e Prática, São Paulo, v.15, n.1, p.105-115, 2013.

NASCIMENTO JUNIOR, J. R. A. et al. Análise das relações entre ansiedade estado e coesão de atletas de handebol. Revista Psicologia: Teoria e Prática, São Paulo, v.18, n.2, p.89-102, 2016a.

NASCIMENTO JÚNIOR, J. R. A. et al. A frequência de jogos como titular e o tempo na equipe são fatores intervenientes na percepção de coesão de grupo no contexto do Futsal de alto rendimento? Revista Inspirar movimento \& saúde, Curitiba, v.16, n.2, p.26-31, 2018.

NASCIMENTO JUNIOR, J. R. A. et al. Propriedades psicométricas do questionário de ambiente de grupo (GEQ) para o contexto do futebol e futsal de alto rendimento.

Revista da Educação Física da UEM, Maringá, v.27, n.1, p.2742, 2016b.

NASCIMENTO JUNIOR, J. R. A.; VIEIRA, L. F. Liderança do técnico e coesão de grupo: um estudo com equipes profissionais de futsal. Revista Brasileira de Ciência e Movimento, Brasília, v.20, n.2, p.84-90, 2012.

NASCIMENTO JUNIOR, J. R. A.; VIEIRA, L. F. Coesão de grupo e liderança do treinador em função do nível competitivo das equipes: Um estudo no contexto do futsal paranaense. Revista Brasileira de Cineantropometria e Desempenho Humano, Florianópolis, v.15, n.1, p.89-102, 2013.

NASCIMENTO JUNIOR, J. R. A. et al. Validação do Questionário de Ambiente de Grupo (GEQ) para a língua portuguesa. Motriz, Rio Claro, v.18, n.4, p.770-782, 2012.

NASCIMENTO JUNIOR, J. R. A. et al. Nível de satisfação do atleta e coesão de grupo em equipes de futsal adulto. Revista Brasileira de Cineantropometria e

Desempenho Humano, Florianópolis, v.13, n.2, p.138-144, 2011. 
PAES, M. J. et al. Frequência, intensidade e direção da ansiedade e sua relação com a coesão grupal em uma equipe de voleibol infanto-juvenil masculina. Revista Brasileira de Psicologia do Esporte, Brasília, v.6, n.3, p.46-56, 2016.

PAGANO, M.; GAUVReAU, K. Princípios de Bioestatística. 2. ed. São Paulo: Pioneira Thomson Learning, 2004.

SEZER, U.; KOCAEKSI, S. Examination of youth athletes' team cohesion and collective efficacy beliefs. Turkiye Klinikleri Journal of Sports Sciences, Istanbul, v.10, n.1, p.1-9, 2018.

SILVEIRA, F. J. T.; OLIVEIRA, L. P. A coesão de grupo realacionada à satisfação das necessidades básicas no esporte em karatecas. Revista Cesumar, Maringá, v.22, n.1, p.7-17, 2017.

TERTULIANO, I. W. et al. Coesão de grupo em categorias de base do futebol. Revista Brasileira de Ciência e Movimento, Brasília, v.27, n.2, p.37-47, 2019.

THOMAS, J. R.; NELSON, J. K.; SILVERMAN, S. J. Métodos de Pesquisa em Atividade Física. 6. ed. Porto Alegre: ArtMed, 2012.

TRIOLA, M. F. Introdução à Estatística. 12. ed. Rio de Janeiro: LTC, 2017.

VERZANI, R. H. et al. Propriedades psicométricas do questionário de ambiente de grupo. Revista Brasileira de Futsal e Futebol, São Paulo, v.11, n.4, p.399-408, 2019.

VIEIRA, L. F. et al. Estado de Humor e Desempenho Motor: um estudo com atletas de voleibol de alto rendimento. Revista Brasileira de Cineantropometria e Desempenho Humano, Florianópolis, v.10, n.1, p.62-68, 2008.

WEINBERG, R. S.; GOULD, D. Fundamentos da psicologia do esporte e do exercício. 6. ed. Porto Alegre: ArtMed, 2017.

WERNECK, F. Z. et al. Características preditoras da escalação de jovens atletas de futsal. Revista Brasileira de Futebol, Viçosa, v.8, n.1, p.43-53, 2015.

XAVIER, G. H. C. et al. Coesão de grupo no Basquetebol: olhares nas categorias de base. Arquivos de Ciências da Saúde da UNIPAR, Umuarama, v.24, n.1, p.53-59, 2020. 